\title{
Specific Absorption Rate Reduction Using Nonlinear Gradient Fields
}

\author{
Emre Kopanoglu, ${ }^{1,2}$ Ugur Yilmaz, ${ }^{1,2}$ Yildiray Gokhalk, ${ }^{1}$ and Ergin Atalar ${ }^{1,2 *}$
}

The specific absorption rate is used as one of the main safety parameters in magnetic resonance imaging. The performance of imaging sequences is frequently hampered by the limitations imposed on the specific absorption rate that increase in severity at higher field strengths. The most well-known approach to reducing the specific absorption rate is presumably the variable rate selective excitation technique, which modifies the gradient waveforms in time. In this article, an alternative approach is introduced that uses gradient fields with nonlinear variations in space to reduce the specific absorption rate. The effect of such gradient fields on the relationship between the desired excitation profile and the corresponding radiofrequency pulse is shown. The feasibility of the method is demonstrated using three examples of radiofrequency pulse design. Finally, the proposed method is compared with and combined with the variable rate selective excitation technique. Magn Reson Med 70:537-546, 2013. (c) 2012 Wiley Periodicals, Inc.

Key words: specific absorption rate; nonlinear gradient fields; high order gradient fields; magnetic resonance imaging

\section{INTRODUCTION}

This study introduces a novel method for reducing the specific absorption rate (SAR) that uses gradient fields with nonlinear variations in space.

To prevent patient hyperthermia or other adverse effects during a magnetic resonance imaging (MRI) examination, the maximum SAR applied to patients has been regulated $(1,2)$. However, reducing the SAR below a certain level may impose restrictions on certain sequence parameters such as the maximum flip angle, the minimum repetition time, and the minimum pulse duration. The SAR varies quadratically with the field strength (3); therefore, restrictions on the sequence parameters become more pronounced, as the field strength increases. Although homogeneous $B_{1}^{+}$fields are important in obtaining diagnostic-quality images, homogeneous fields can only be obtained with additional increases in the SAR. Because the restrictions imposed on the SAR hamper the imaging performance, reduction of the SAR while maintaining the levels of other imaging parameters has become an active research area in the field.

\footnotetext{
${ }^{1}$ National Magnetic Resonance Research Center (UMRAM), Bilkent University, Bilkent, Ankara, Turkey.

${ }^{2}$ Department of Electrical and Electronics Engineering, Bilkent University, Bilkent, Ankara, Turkey.

${ }^{*}$ Correspondence to: Ergin Atalar, Ph.D., UMRAM, Cyberplaza, Block C, 2nd Floor, Cyberpark, Bilkent University, Bilkent, Ankara 06800, Turkey. E-mail: ergin@ee.bilkent.edu.tr

Received 21 March 2012; revised 5 July 2012; accepted 7 August 2012.

DOI 10.1002/mrm.24478

Published online 17 September 2012 in Wiley Online Library (wileyonlinelibrary.com).

There is a trade-off between $B_{1}^{+}$-homogeneity and the SAR at all field strengths. In the high-field regime, $B_{1}^{+}$inhomogeneities are more severe because of the increasing interference effects due to the wavelength and field attenuation caused by tissue conductivity. Therefore, various techniques have been devised to mitigate $B_{1}^{+}$-inhomogeneities. Examples of such techniques include $B_{1}^{+}$-shimming and multidimensional excitation pulses. The $B_{1}^{+}$-shimming methods (4) use coils with distinct spatial field variations and increase the homogeneity through separate adjustment of the signal amplitude and the phase for each coil. However, such methods increase whole-body and local (especially in regions close to the individual transmit coil elements) SAR values. Multi-dimensional pulses (5-9) increase the homogeneity by specifying the variation of the excitation profile in three dimensions. However, because such pulses are more condensed than the conventional excitation pulses, obtaining the same flip angle without increasing the pulse duration requires a higher SAR. In contrast, the effects of inhomogeneity are less severe in the low-field regime; therefore, approaches designed to reduce inhomogeneity in this regime are mostly limited to coil design. Although the "stubby" birdcage coil (10) was designed to optimize the signal-to-noise ratio along its axis, the coil generates a nonhomogeneous $B_{1}^{+}$field. Hence, longer birdcage coils are used instead. However, these longer coils expose a larger volume of the subject to electromagnetic waves, increasing the whole-body SAR (but not necessarily local SAR). The trade-off between $B_{1}^{+}$homogeneity and the SAR is not a problem limited to the high-field regime but represents a major problem at all field strengths.

Arguably, the most well-known approach for decreasing the SAR is variable-rate selective excitation (VERSE) (11). With time-varying gradient waveforms instead of the conventional trapezoidal waveforms, the VERSE algorithm modifies the amplitude of the radiofrequency (RF) pulse to decrease the whole-body SAR while keeping the spatial variations of the SAR and the flip angle unaltered. Although this approach can significantly reduce the SAR, the performance of this method is limited when the gradient waveforms are already restricted by the limitations on the slew rate or the gradient amplitude. Furthermore, the off-resonance performance of the VERSE technique is rather poor, because the time varying gradient waveforms cause blurring.

It is known that the gradient fields are not perfectly linear and that these nonlinearities cause curved slices and warped images. To correct for such effects, many recent studies have focused on measuring these nonlinearities using field probes (12) or preceding measurement scans (13). As an alternative, several investigators have 
exploited the nonlinearity of the gradients to their advantage by obtaining curved slices (14-17) and nonuniform resolution (18-22). It has been shown that when nonlinear gradient fields (NLGFs) are used, the adiabatic condition in flow-driven arterial spin labeling measurements could be achieved with smaller RF pulse amplitudes and as a result, lower SAR values (23). However, to the best of our knowledge, no research has been conducted that demonstrates the effect of NLGFs on the RF pulse shape toward reducing the SAR.

In this study, we propose using gradient fields with nonlinear variations in space to reduce the whole-body SAR without changing the spatial distribution of the SAR (24). To reduce the complexity of the problem, we first demonstrate the technique on two one-dimensional (1D) excitation cases. In a third example, we study the effect of an NLGF on the SAR in a multidimensional excitation scheme. Furthermore, we compare and combine the proposed technique with the VERSE algorithm.

\section{THEORY}

Conventional MRI methods use fields that vary linearly in space for excitation and encoding, also known as gradient fields. In contrast, the higher-order fields, which are used for shimming the static magnetic fields, are widely called shim fields. In this text, we will refer to the former as linear gradient fields (LGFs) and to the latter as NLGFs, to signify their respective roles in the encoding process.

The SAR depends on many factors, including the RF coil, the flip angle, the pulse duration, and the pulse shape. This article focuses on altering the pulse shape using NLGFs. To emphasize this effect on the SAR, we define the normalized SAR (nSAR) as follows:

$$
\mathrm{nSAR} \triangleq \int_{-1 / 2}^{1 / 2}|b(s)|^{2} \mathrm{~d} s
$$

where $b(s)$ denotes the normalized RF pulse (relative to unit duration and unit integral), as defined by the following equation:

$$
b(t / \tau) \triangleq \frac{\gamma \tau}{\alpha} B_{1}(t)
$$

where $\gamma$ denotes the gyromagnetic ratio, and $B_{1}(t), \tau$, and $\alpha$ denote the envelope, the duration, and the flip angle of the $\mathrm{RF}$ pulse, respectively. The minimum value for the nSAR is one, which is the value that holds for a rectangular pulse.

First, the relationship between the nSAR and the excitation profile will be formulated for 1D gradient fields and objects for simplicity. We define the excitation profile as the transverse component of the magnetization normalized by the magnetization density and denote this unitless quantity as $m(z)$. It is known that at the small-tip-angle regime, the $\mathrm{RF}$ pulse that approximately excites the desired excitation profile can be expressed by the following equation (25):

$$
B_{1}(t)=-i \frac{G}{2 \pi} M\left(\frac{\gamma}{2 \pi} G t\right)
$$

where $G$ denotes the amplitude of the LGF, and $M(k)$ denotes the Fourier transform of the excitation profile (i.e., the excitation k-space), where $k=\frac{\gamma}{2 \pi} G t$ and $i$ is the imaginary unit; in this equation, a complete refocusing is assumed. In practice, the RF pulses are limited in time using apodization functions. Because such a function complicates the discussion, in this article, we simply truncate the RF pulses to a duration of length $\tau$. In this case, the normalized RF pulse can be expressed by the following equation:

$$
b(s)=\frac{M\left(\frac{\gamma}{2 \pi} G \tau s\right)}{\left|\int_{-1 / 2}^{1 / 2} M\left(\frac{\gamma}{2 \pi} G \tau s^{\prime}\right) \mathrm{d} s^{\prime}\right|}
$$

It can be observed from Eqs. 1 and 4 that the nSAR depends on the excitation profile.

When an NLGF is used, the dependence of the SAR on the excitation profile is altered. Instead of the gradient field $G z$, let us assume a nonlinear field $G_{W} W$ where $w=f(z)$ denotes the variation of the field in space is used, and $w$ is defined as a nonlinear coordinate variable. When the transformation from $z$ to $W$ is bijective, the inverse function, $f^{-1}(w)$, exists. In this case, the excitation profile can be expressed in terms of the nonlinear coordinate: $m(z)=m\left(f^{-1}(w)\right)=m_{\mathrm{n}}(w)$, and the normalized RF pulse can be expressed in terms of the Fourier transform of $m_{n}(w)$, $M_{\mathrm{n}}(\cdot)$ as shown in the following formula:

$$
b_{\mathrm{n}}(s)=\frac{M_{\mathrm{n}}\left(\frac{\gamma}{2 \pi} G_{W} \tau s\right)}{\left|\int_{-1 / 2}^{1 / 2} M_{\mathrm{n}}\left(\frac{\gamma}{2 \pi} G_{W} \tau s^{\prime}\right) \mathrm{d} s^{\prime}\right|}
$$

Figure 1 demonstrates how the RF pulse changes when an NLGF with $f(z)=z-10^{-3} \sin (2 \pi z / 0.032)$ variation (all distances are in meters) is used. It is known that the slope of the gradient field affects the thickness of the excitation profile. For NLGFs, because the slope varies with position, the excitation profile is distorted. To correct this distortion, the excitation k-space needs to be distorted in a compensatory fashion that corresponds to another RF pulse shape (Fig. 1) and a different SAR value; for this example, the nSAR is reduced from 2.16 to 1.85. As a result, the SAR changes when an NLGF is used.

It is known that unless the region of interest is not extremely narrow in at least one direction, a 1D NLGF is not realizable; such a field would display a similar variation along at least another direction. Therefore, the above formulation will be expanded to three-dimensional (3D) objects and multidimensional RF excitation pulses. Although the multispoke approach (9) is used in this article, these formulations can be trivially modified to fit other schemes.

As in the 1D case, the transformation from the linear coordinate system, $\mathbf{x}$ (with spatial variables $x, y$, and $z$ ) to the nonlinear coordinate system, $\mathbf{u}$ (with spatial variables $u, v$, and $w$ ) can be written as $\mathbf{u}=\mathbf{f}(\mathbf{x})$, where $\mathbf{f}(\mathbf{x})$ denotes the spatial variation of the NLGFs. If the NLGFs are bijective, then the desired excitation profile, $m(\mathbf{x})$, can be expressed as $m_{\mathrm{n}}(\mathbf{u})=m\left(\mathbf{f}^{-1}(\mathbf{u})\right)$. Hence, the $p$ th spoke of the normalized RF pulse can be expressed as

$$
b_{\mathrm{n} p}(s)=\frac{M_{\mathrm{n}}\left(k_{u p}, k_{v p}, \frac{\gamma}{2 \pi} G_{W} \tau s\right)}{\left|\sum_{p=1}^{P} \int_{-\tau_{p} / 2 \tau}^{\tau_{p} / 2 \tau} M_{\mathrm{n}}\left(k_{u p}, k_{v p}, \frac{\gamma}{2 \pi} G_{W} \tau s^{\prime}\right) \mathrm{d} s^{\prime}\right|}
$$

where $M_{\mathrm{n}}\left(\mathbf{k}_{\mathrm{n}}\right)$ is the Fourier transform of $m_{\mathrm{n}}(\mathbf{u})$ and $\mathbf{k}_{\mathrm{n}}=$ $\left[\begin{array}{lll}k_{u} & k_{v} & k_{w}\end{array}\right]$. In Eq. 6, $p$ is the spoke index, $P$ is the total 

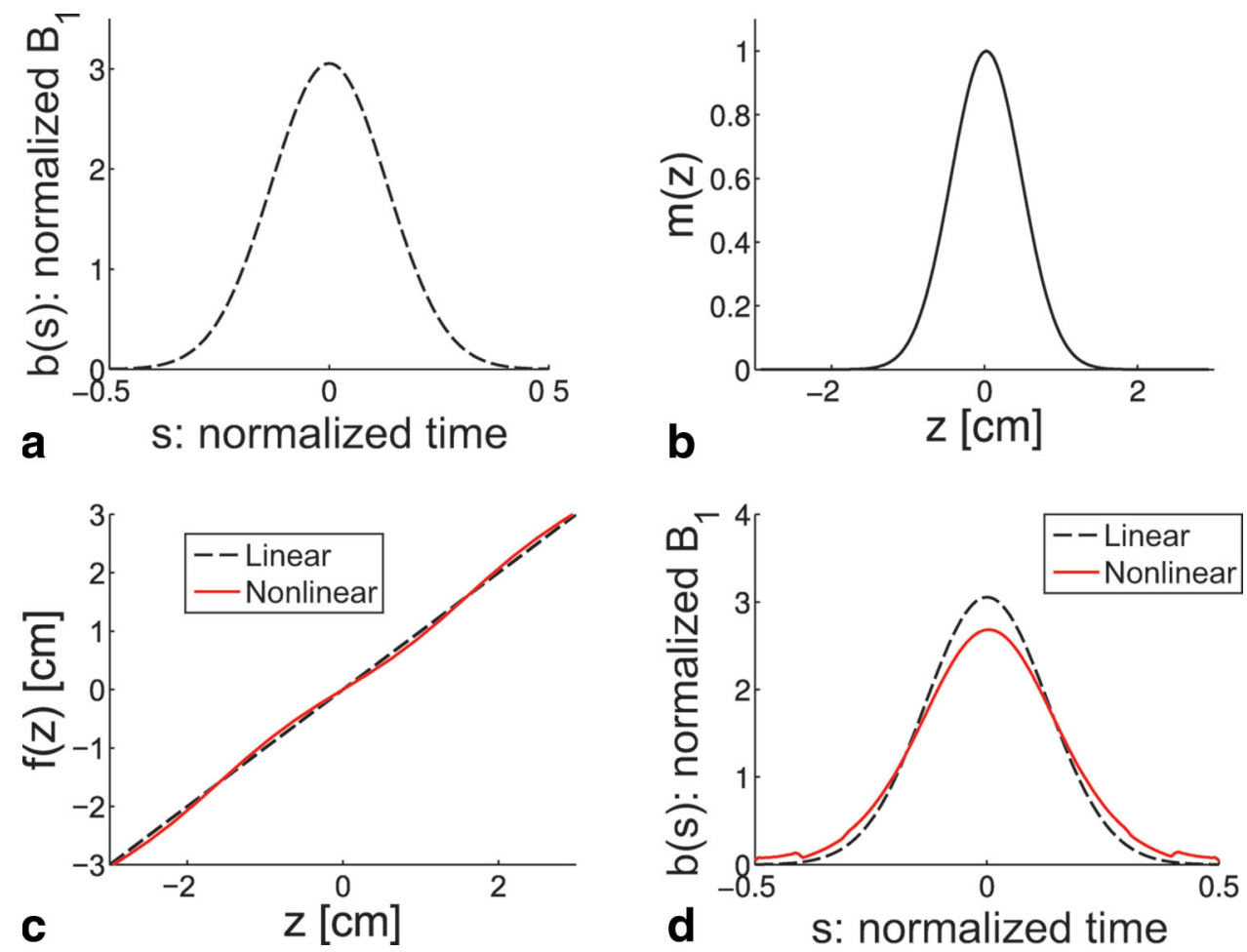

FIG. 1. An example demonstrating the effect of using an NLGF on a normalized RF pulse. a: An apodized sinc pulse with a T-BW product of 2 is selected as the reference solution. b: The excitation profile of the apodized sinc pulse in the presence of a perfect LGF. c: The variation of the NLGF in space compared with the LGF. d: The RF pulse that obtains the desired profile in the presence of the NLGF is compared with the apodized sinc pulse. [Color figure can be viewed in the online issue, which is available at wileyonlinelibrary.com.]

number of spokes, $\tau_{p}$ is the duration of the $p$ th spoke and $\tau=\sum_{p=1}^{P} \tau_{p}$ is the total pulse duration. It should be noted that, because this is a spoke excitation scheme, multiple $\mathrm{RF}$ segments that are in the $k_{W}$-direction, positioned at $\left(k_{u p}, k_{v p}\right)$ locations are used. Finally, the nSAR can be calculated by normalizing the total RF waveform by $\tau$. When $\mathbf{u}, G_{W}$, and $M_{\mathrm{n}}(\cdot)$ are replaced with $\mathbf{x}, G_{z}$, and $M(\cdot)$, Eq. 6 reduces to the linear case, resulting in $b(s)$. Although the general formulations of these equations are similar, the excitation k-space is affected by the gradient nonlinearity when $M(\mathbf{k})$ is replaced by $M_{\mathrm{n}}\left(\mathbf{k}_{\mathrm{n}}\right)$. Hence, the SAR is altered when an NLGF is used.

When the nonlinear field is nonbijective, special care must be taken when applying the above formulation. In this case, the same field values are observed at multiple locations in space, meaning that the amount of excitation (i.e., the flip angle) will be the same at those positions. Therefore, whether or not the above formulation can be used directly depends on the desired excitation profile. We will now investigate such cases and provide simple 1D examples for each case:

i. In special cases, the desired excitation profile can be compatible with the nonbijective NLGF. For example, an excitation profile symmetric around $z=0$ can be obtained using a $z^{2}$ field. In this case, no special treatment is necessary.

ii. In other cases, the field can be made compatible with the desired profile by combining it with other gradient fields, for example, a symmetric profile around $z=z_{0}$, where $z_{0} \neq 0$ cannot be realized with a $z^{2}$ field. However, a $z^{2}-2 z z_{0}$ field is compatible with the profile and can be obtained with the simultaneous use of an LGF with the NLGF.

iii. If no combination of the available fields yields a compatible field distribution, then the excitation profile can only be approximately generated using nonbijective fields. However, in such a case, the residual part of the profile can be generated using a set of bijective gradient fields. For example, a profile that is not symmetric can be decomposed into symmetric and antisymmetric components; the former can be obtained using the $z^{2}$ field, whereas the latter can be excited using an LGF.

As it can be observed, even nonbijective fields can be used to obtain the desired excitation profile when integrated with the proper combination of LGFs.

When NLGFs are used for excitation, the excitation kspace, and hence the SAR, are modified. Depending on the nonlinearity of the field and the excitation profile, the SAR may decrease or increase. However, if LGFs are still available, the SAR-optimal solution will use the LGFs in the worst case. Therefore, when NLGFs are added to the set of available fields, the SAR may only be reduced.

\section{METHODS}

The effect of NLGFs on excitation and the SAR is demonstrated with three example cases. To highlight such effects, 
the first two examples are 1D; the third is 3D to demonstrate how a realistic case can be handled. The simulations are performed in Matlab (The Mathworks Inc., Natick, MA), and the experiments are performed using a 3T scanner (MAGNETOM Trio a Tim System, Siemens Healthcare, Erlangen, Germany). As a descriptor of excitation efficiency, the root mean squared error (RMSE) between the desired and obtained profiles is calculated (6). The RMSE and nSAR values will be reported in "Results" section.

\section{(Case 1) 1D: Pulse Design for a Given Nonlinear Field}

In this example, the effect of a slight nonlinearity on the SAR is investigated using the field distribution given in Figure 1. An apodized sinc pulse with no side lobes (timebandwidth product: 2), the default RF pulse in the basic gradient echo pulse sequence obtained from the manufacturer of the scanner, is used as the reference solution. Using the excitation profile of the reference solution (Fig. 1), the RF pulse for the NLGF case was designed.

To realize the desired NLGF experimentally, a coil with a 2.4-cm diameter is designed on a polyoxymethylene cylinder using the target-field method (26). The required current distribution along the $z$-axis is sampled at intervals of $4-\mathrm{mm}$ and truncated to a total length of $6.4 \mathrm{~cm}$. The corresponding field is simulated and given in Figure 2. Note that although truncation causes some deviations in the field, these deviations are outside the full-width-half-max (FWHM) of the desired excitation profile and therefore, only slight contractions are expected outside the FWHM.

In the experiments, a cylindrical water phantom with a 1.4-cm diameter is used. To observe the excitation profile, the readout is performed in the excitation direction. To drive the custom-made coil, the optically isolated $x$ gradient waveform generated by the scanner is amplified using an audio amplifier (TonyLee Dj 451, JCLEON, PRC; maximum power, $450 \mathrm{~W}$ ). The maximum required current was $\approx 0.1 \mathrm{~A}$. To limit the waveform distortion to below $2 \%$, $20 \Omega$ resistance is used in series with the amplifier. During the experiments, the amplifier of the $x$-channel was turned off to keep the excitation direction parallel to the $z$-axis. Therefore, the $B_{0}$-shimming is compromised, but the effect of the intravoxel signal cancelation was acceptable for the small phantom used in the experiments. In the experiments, a gradient echo sequence is used with the following parameters: flip angle, $15^{\circ}$; RF duration, $1.25 \mathrm{~ms}$; pulse repetition time, $9.1 \mathrm{~ms}$; echo time, $4.9 \mathrm{~ms}$; excitation gradient amplitude, $7.4 \mathrm{mT} / \mathrm{m} ; \mathrm{FOV}_{y}$ and $\mathrm{FOV}_{z}, 116 \mathrm{~mm}$ and acquisition matrix, $128 \times 256$.

\section{(Case 2) 1D: Field Design for Optimal SAR Pulses}

Although a rectangular pulse is optimal in terms of nSAR, its excitation profile is not sufficiently selective; this promotes the use of other RF envelopes that have better selectivity—such as apodized sinc pulses—at the expense of higher nSAR (Fig. 3). It is known that by changing the slope of the gradient field, the excitation profile can be contracted or expanded. Therefore, by designing a field with a low slope in the excitation region and high slopes at the boundaries, the main lobe of the excitation profile of a rectangular pulse can be expanded, and the side lobes of the
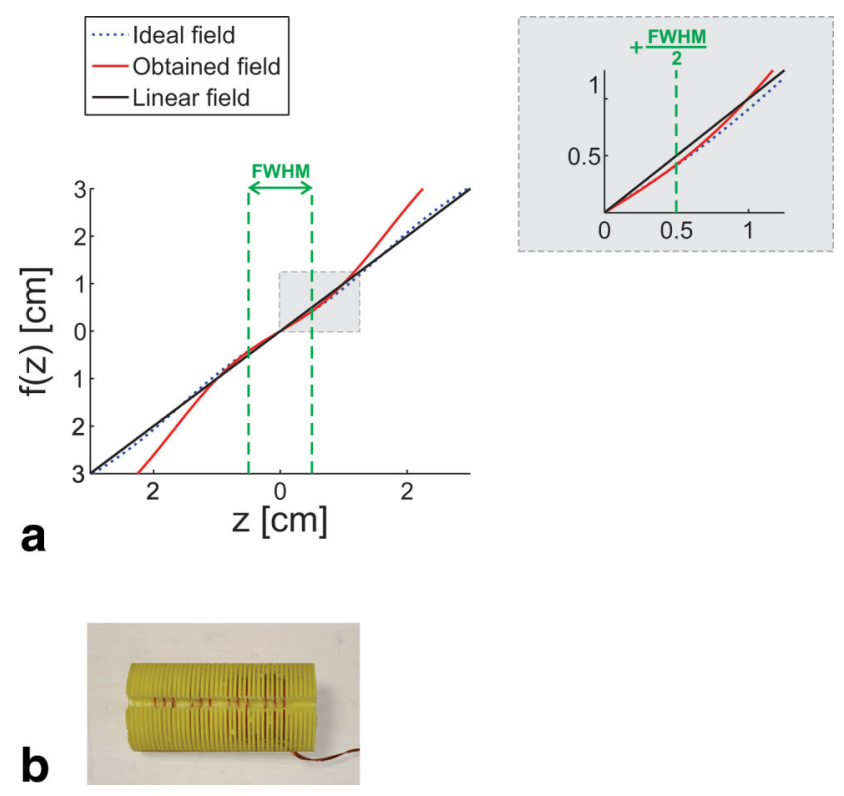

FIG. 2. The effect of truncating the required current distribution on the resulting field and the coil produced using the truncated distribution. a: The field realized when the current distribution is truncated to $6.4 \mathrm{~cm}$ is compared with the ideal and the linear fields: a close-up version of $f(z)$, indicating half of the FWHM shows that the deviation of the realized field from the ideal is negligible inside the FWHM (top-right corner). b: The gradient coil wound on a polyoxymethylene cylinder. Winding separation: $4 \mathrm{~mm}$ and winding diameter: $2.4 \mathrm{~cm}$. [Color figure can be viewed in the online issue, which is available at wileyonlinelibrary.com.]

excitation profile can be contracted, yielding enhancement in selectivity. Although the optimal NLGF would have infinite slopes at the boundaries, a physically realizable field that approximates this ideal field with the following expression

$$
f(z)=z+0.47 \frac{z}{1+(z / 0.09)^{16}}-1.47 \frac{z}{1+(z / 0.03)^{16}},
$$

where all distances are in meters, is designed using the target field method (26). To realize the field experimentally, a coil is wound on a cylinder similar to that used in "Case 1 ", (Fig. 3). However, because the field magnitude in this case is higher, the realized field can be measured using MRI by feeding the coil with the phase-encoding signal during a gradient echo image and by extracting the field of the coil from the distortion in the image (Fig. 3). The measured field is used as $w=f(z)$ in the simulations. It should be noted that, the number of turns is increased without changing the required current to attain the higher field magnitudes. The same RMSE definition, experimental setup and sequence parameters are used as in Case 1.

\section{(Case 3) 3D: Inhomogeneity Correction Using Multidimensional Pulses}

In the third example, which is 3D, the effect of NLGFs on the SAR is demonstrated in an SAR-intensive multispoke excitation scheme. Multidimensional pulses are commonly 


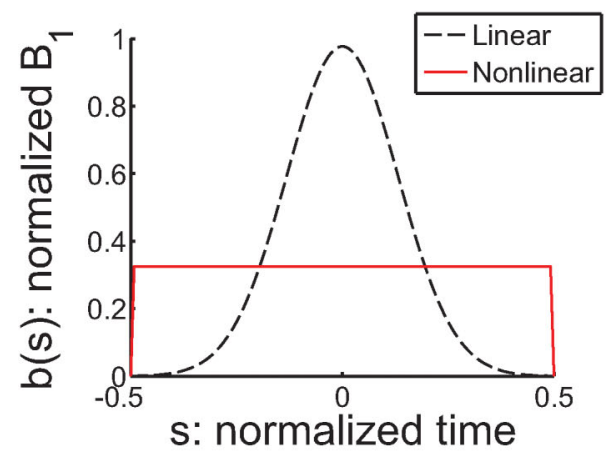

a

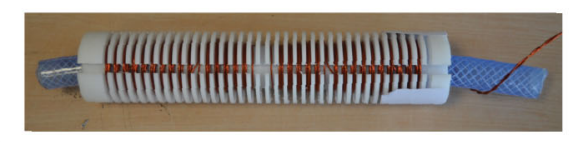

C

FIG. 3. An NLGF designed to obtain a selective excitation profile when a rectangular RF pulse is applied, and the resulting excitation profile compared with the profile of an apodized sinc pulse. a: The normalized apodized sinc (T-BW product: 2) and rectangular pulses. b: The ideal and the designed fields to obtain a better selective excitation profile with the rectangular RF pulse. c: The gradient coil wound on a polyoxymethylene cylinder, and the thin tubular phantom used in the experiment. Coil length: $20 \mathrm{~cm}$, winding separation: $5 \mathrm{~mm}$, and winding diameter: $2.4 \mathrm{~cm}$. d: The generated field extracted from the distortion in the resulting gradient-echo image by feeding the coil with the phase-encoding signal, compared with the designed field. [Color figure can be viewed in the online issue, which is available at wileyonlinelibrary.com.]

used for tailoring the excitation profile in three dimensions for $B_{1}^{+}$-inhomogeneity correction (6-8). Although $B_{1}^{+}$inhomogeneities due to wavelength effects become more pronounced in the ultrahigh-field regime, they are still evident even at $3 \mathrm{~T}$, because the wavelength is $27 \mathrm{~cm}$ (assuming a relative permittivity of 80 ). In this example, we set our desired excitation profile to be a slab with a thickness of $12 \mathrm{~cm}$. However, when a conventional RF pulse is transmitted with the body coil, nearly $30 \%$ signal variation can be observed in a 12-cm field of view (FOV, Fig. 4). To mitigate this inhomogeneity effect, multispoke RF pulses are designed.

To demonstrate the effect of using NLGFs on the SAR, a slight nonlinearity is introduced to the $z$-gradient using a Maxwell pair (Fig. 5). Two multispoke pulses were designed using the three LGFs and using the NLGF together with the $x$ - and $y$-gradient fields. First, by dividing the ideal profile by the inhomogeneity (Fig. 4), the desired profile is obtained. It should be noted that the axial cross section of the ideal profile is slightly smaller than that of the phantom. For the regions outside the ideal profile, no correction is performed. Second, the excitation k-space of the desired profile is obtained using Fourier transform. The excitation $\mathrm{k}$-space is sampled by retaining the regions that contain the highest power and discarding the rest. By tracing the sampled excitation k-space using the gradient waveforms,
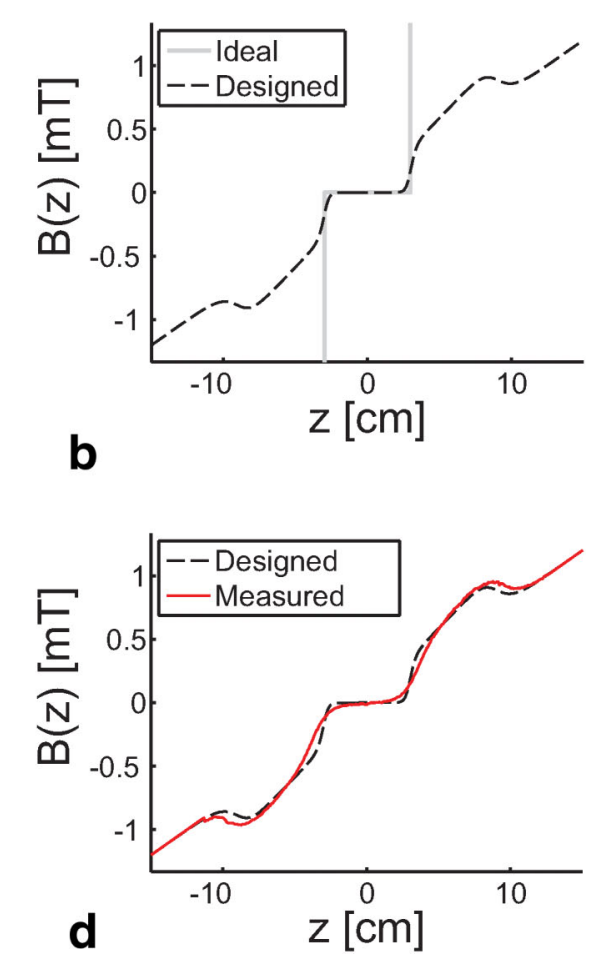

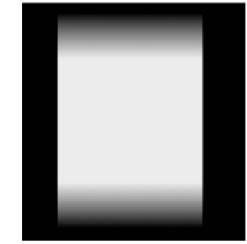

a

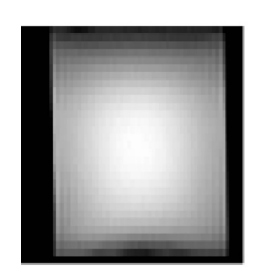

b
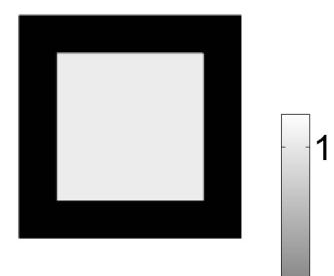

0.5
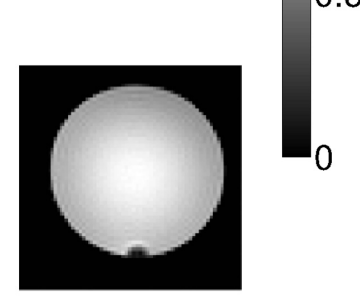

FIG. 4. The desired and obtained excitation profiles when a conventional excitation pulse is applied. a: The desired excitation profile, a slab with a $9-\mathrm{cm}-$ long homogeneous region and $1.5-\mathrm{cm}$ long transition regions along the $z$-direction; the simulation phantom, measuring $10 \times 10 \times 30 \mathrm{~cm}^{3}$. b: The presence of central brightening effect in the resulting phantom (Model No: 8624186 K2285, Siemens, Germany; diameter: $12 \mathrm{~cm}$, length: $25 \mathrm{~cm}$ ) images when a conventional excitation pulse is applied. Coronal images (left), FOV: $15 \times 16 \mathrm{~cm}^{2}$. Axial images (right), FOV: $15 \times 15 \mathrm{~cm}^{2}$. 

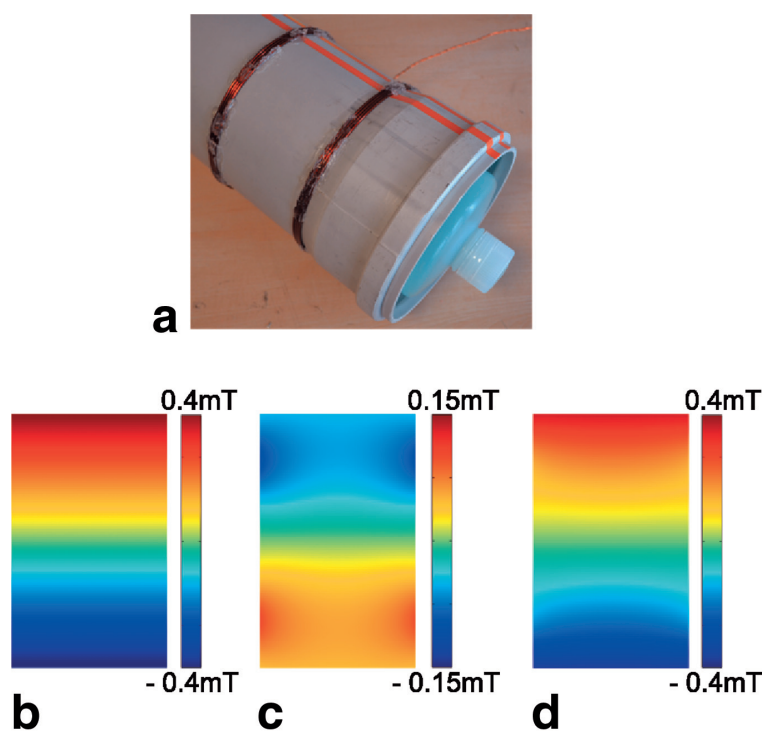

FIG. 5. The produced Maxwell coil and the simulated LGF and NLGFs. All fields are given on the coronal plane with FOV: $10 \times 16 \mathrm{~cm}^{2}$ and cylindrical symmetry. a: The Maxwell coil, wound on a plastic cylinder with 16-cm diameter (two opposite windings of four turns each), and the Siemens phantom used in the experiments. b: The z-gradient field. c: The field generated by the Maxwell pair. d: The NLGF obtained using the Maxwell pair together with the z-gradient.

the RF pulse is obtained. Although the scanner used for the experiments can support a $40 \mathrm{mT} / \mathrm{m}$ field strength, the spoke-gradient field strength was limited to $4.7 \mathrm{mT} / \mathrm{m}$ to assure that the excitation k-space is sampled sufficiently dense. For all gradients, a $120 \mathrm{mT} / \mathrm{mms}$ slew rate limit is used. Instead of limiting the total number of spokes, the total RF pulse duration is limited to $2.5 \mathrm{~ms}$. For the NLGF case, the desired profile is first mapped to the nonlinear coordinate system, and the corresponding excitation $\mathrm{k}$-space is subsequently obtained and sampled.

To simulate the expected excitation profile, the inverse Fourier transform is applied to the sampled excitation kspace. Next, the effect of the inhomogeneity is applied to the resulting excitation profile. It should be noted that when the NLGF is used, the profile is mapped to Cartesian coordinates before applying the inhomogeneity. The flip angle of the expected excitation profile is adjusted to minimize the RMSE in the FOV with respect to the ideal profile. Because the transition regions are encoded but discarded in slab imaging schemes, these regions are not included in the error calculations.

In the experiments, 3D encoding is performed with readout, phase, and section encoding along the $x^{-}, y^{-}$, and $z$-directions, respectively. The experimental setup is similar to the previous two cases, but there are three differences: (i) the maximum current required was $\approx 1.25 \mathrm{~A}$; (ii) because $3 \mathrm{D}$ encoding is performed, the $x$-gradient cannot be disconnected; therefore, the $z$-gradient waveform is used for driving the custom coil; and (iii) because the custom coil distorts the field, it should be switched off during encoding; therefore, a mechanical relay is inserted before the audio amplifier. To account for the RF pulse duration and to facilitate proper switching of the relay, an echo time of $10 \mathrm{~ms}$ and a pulse repetition time of $100 \mathrm{~ms}$ are used. The other parameters are set to the following values: FOV, $15 \times 15 \times 16 \mathrm{~cm}^{3}$; acquisition matrix, $64 \times 64 \times 32$; flip angle, $15^{\circ}$. For consistency, both the computational and the experimental results are resampled to $128 \times 128 \times 96$ matrices using linear interpolation.

\section{Implementation of VERSE}

To observe the effect of the VERSE algorithm on the SAR, we increased the gradient amplitude limit by 10, 25, and $100 \%$ while keeping the slew rate limit constant. It should be noted that, the last case is slew-rate limited rather than amplitude limited. An iterative approach is used for the VERSE algorithm by reducing the maximum RF threshold by $20 \%$ with each iteration and by increasing the number of iterations until the total RF duration is again $2.5 \mathrm{~ms}$.

\section{RESULTS}

\section{(Case 1) 1D: Pulse Design for a Given Nonlinear Field}

For the RF pulses shown in Figure 1, the computational and experimental excitation profiles are given in Figure 6. The nSAR of the linear case is 2.16 , whereas the nSAR is reduced by $15 \%$ to 1.85 in the nonlinear case. The excitation profile for the NLGF case has small oscillations outside
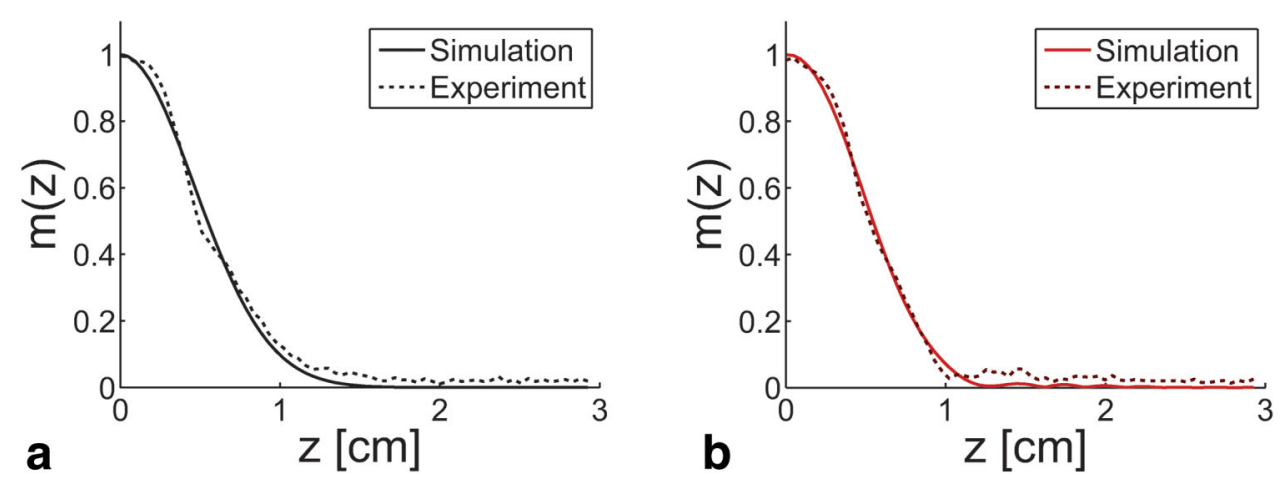

FIG. 6. Comparison of the excitation profiles. a: The profile for the linear case. b: The profile for the nonlinear case. (Due to the symmetry of the profiles, only $z>0$ halves are given.) [Color figure can be viewed in the online issue, which is available at wileyonlinelibrary.com.] 

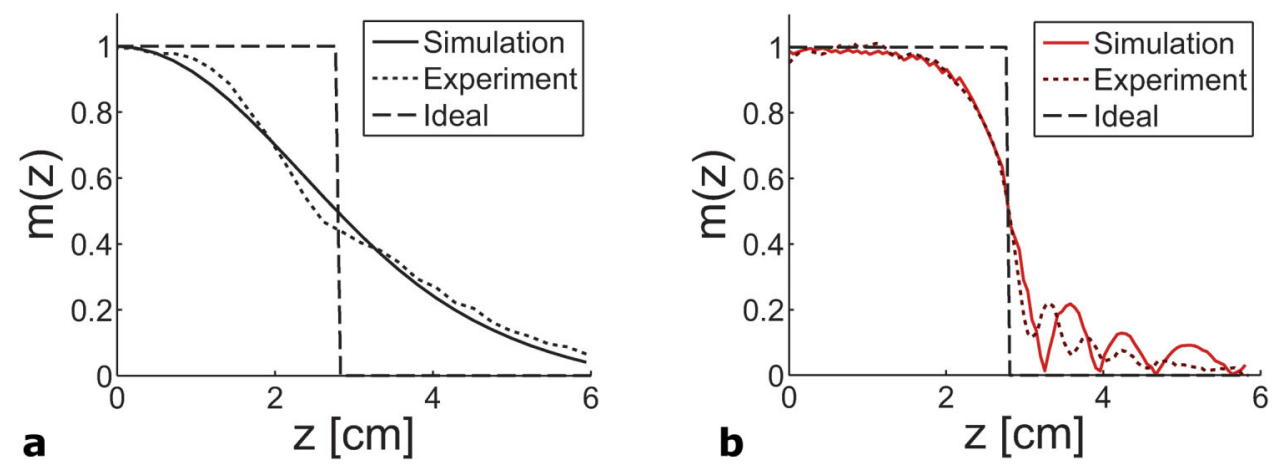

FIG. 7. Comparison of the excitation profiles. a: The profile of the apodized sinc pulse in the presence of the LGF. b: The profile of the rectangular pulse in the presence of the NLGF. In the simulations, the NLGF measured using MRI is used. (Due to the symmetry of the profiles, only $z>0$ halves are given.) [Color figure can be viewed in the online issue, which is available at wileyonlinelibrary.com.]

the main lobe, which causes an RMSE of $0.5 \%$ in the simulations. As expected, slight contractions occur outside the FWHM due to the truncation of the current distribution to $6.4 \mathrm{~cm}$, which increases the RMSE to $1.2 \%$. In the experiments, the RMSE between the LGF and NLGF cases is $2.9 \%$. The RMSEs between the simulations and the experiments are $3.1 \%$ (for the linear case) and $3.4 \%$ (for the nonlinear case). It can be observed that even with a slight nonlinearity, the SAR can be reduced without affecting the excitation profile.

\section{(Case 2) 1D: Field Design for Optimal SAR Pulses}

When the rectangular RF pulse is used (Fig. 3), the nSAR is reduced by $54 \%$ (from 2.16 to 1 ). The RMSEs between the computational and experimental profiles (Fig. 7) are $3.4 \%$ (for the linear case) and $4.9 \%$ (for the nonlinear case). When the profiles are compared with the ideal profile (Fig. 7), the error values for the linear case are $24.5 \%$ in the simulations and $25.6 \%$ in the experiments; for the nonlinear case, the error values are $13.2 \%$ in the simulations and $12.0 \%$ in the experiments. In this example, it is clear that the excitation profile obtained using the rectangular pulse more closely resembles the ideal profile than the reference solution. To obtain better selective profiles in the linear case, the time-bandwidth (T-BW) product of the sinc pulse can be increased at the expense of a higher nSAR. For T-BW values of 4,6 , and 8 , the RMSE drops to $21.7,17.5$, and $15.2 \%$, respectively, whereas the nSAR increases to 3.1 , 5.2 , and 7.2 , respectively. For a $\mathrm{T}-\mathrm{BW} \approx 11$, the RMSE can be reduced to $13.2 \%$, as in the nonlinear case. However, this T-BW value also results in an increase of the nSAR to 9.8. Therefore, in contrast to the LGFs, NLGFs can significantly reduce the nSAR while obtaining better selective excitation profiles.

(Case 3) 3D: Inhomogeneity Correction Using Multidimensional Pulses

Without any correction, the central brightening effect causes an RMSE of $16 \%$ in the excitation profile compared with the ideal profile (Fig. 4). When the multispoke RF pulse designed for the linear case is used, the RMSE is reduced to $4 \%$ (Fig. 8); however, the nSAR of the RF pulse is 110.9. For the NLGF case, the nSAR is reduced by $26 \%$ to 82.6, although the RMSE is slightly increased to $5 \%$.

When the VERSE algorithm is implemented by increasing the maximum gradient amplitude by 10,25 , and $100 \%$, the nSAR values are reduced to 70,40 , and 25.5 , respectively, for the LGF case and 42.6, 24.2, and 15.3, respectively, for the NLGF case (Fig. 9). Hence, the nSAR is $39-40 \%$ lower for the NLGF case.

\section{DISCUSSION AND CONCLUSIONS}

In this article, a new SAR reduction method is introduced that uses NLGFs. To compare various RF pulses designed with LGFs and NLGFs, the nSAR is defined. Using three example cases, SAR reductions between 15 and 54\% are shown in both simulations and experiments.

Conventional MRI strategies assume perfectly LGFs; therefore, the reduction of any imperfections in the LGFs has been studied extensively. However, some nonlinearities always exist. For this reason, many algorithms that correct the effects of such nonlinearities on the images are implemented in commercially available scanners. As demonstrated, such nonlinearities may be useful in terms of the SAR. In the first example case, a slight sinusoidal nonlinearity was assumed to exist with the LGF, and the SAR was reduced by $15 \%$.

The commonly used selective excitation pulses, such as apodized sinc envelopes, are far from optimal in terms of the SAR. In fact, we demonstrate that such pulses may have more than twice the minimum SAR value, which holds for a rectangular pulse. However, the excitation profile of a rectangular pulse does not provide sufficient selectivity, limiting its use in MRI. With NLGFs, we demonstrate that the excitation profile of a rectangular pulse can be transformed into a selective profile that also yields the lowest possible SAR.

Multidimensional pulses significantly increase the SAR. The nSAR of the multispoke pulse designed with the LGFs was more than two orders of magnitude higher than that of a rectangular pulse. However, the SAR can be significantly decreased with NLGFs. In the third example case, the SAR was reduced by approximately 26 to $40 \%$. It should be noted that the NLGF used for this example was selected for its simplicity, meaning that it does not imply a limit 


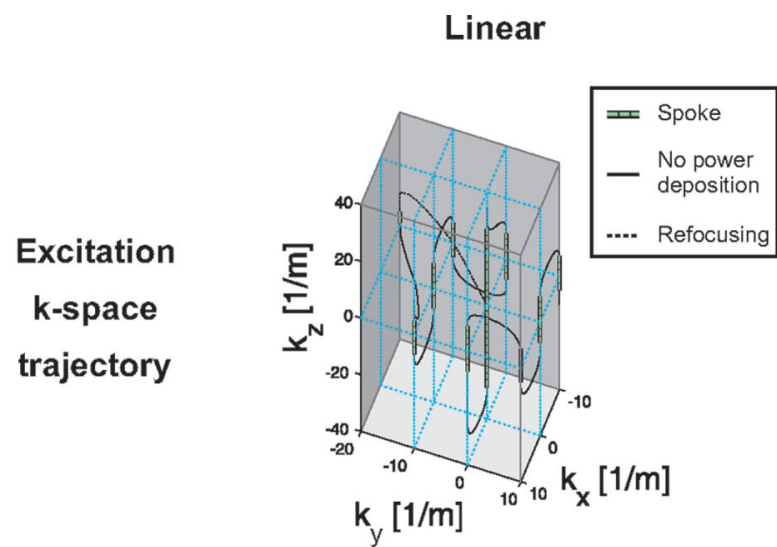

RF pulse
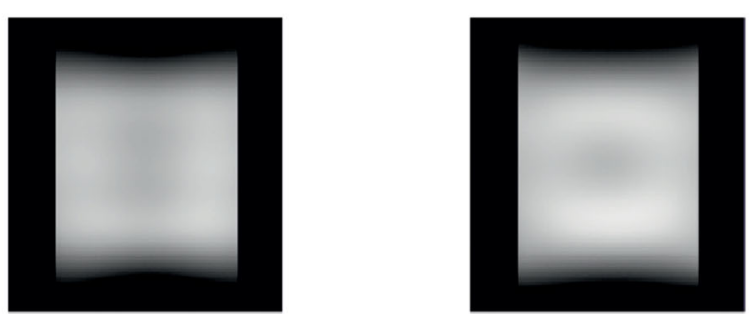

\section{Simulation \\ coronal \\ plane}

\section{Simulation \\ axial plane}
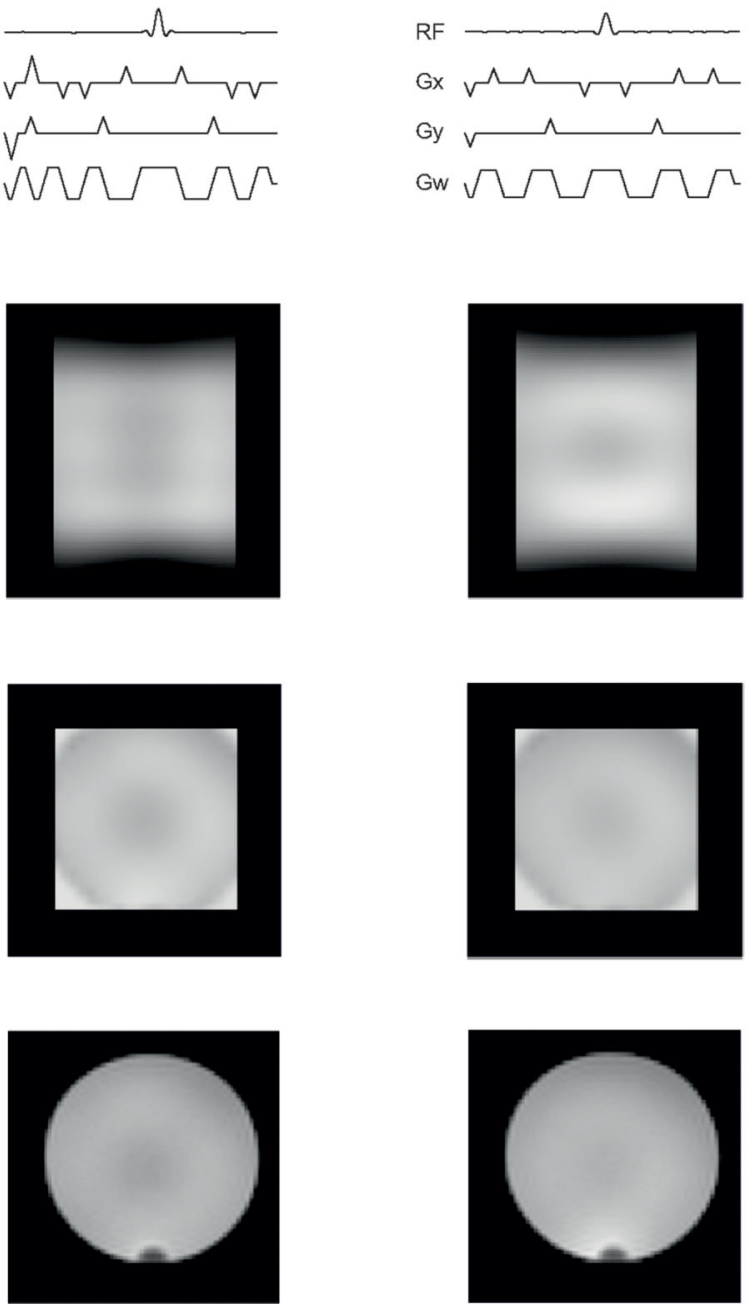

Nonlinear

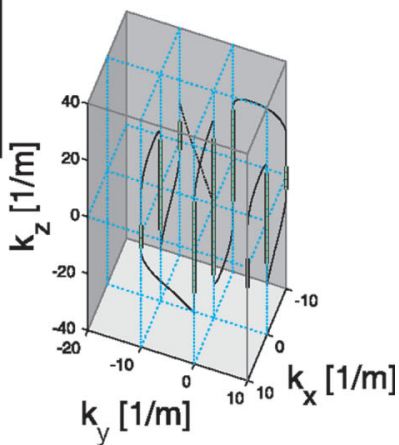

$k_{y}[1 / m]$

\section{Experiment \\ axial plane}

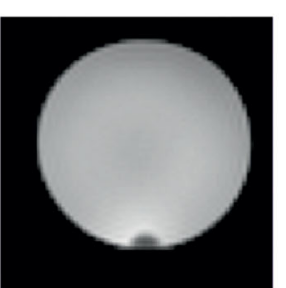

FIG. 8. Comparison of the excitation k-space trajectories, RF pulses and the corresponding excitation profiles obtained for the linear and nonlinear cases. Because of finite gradient slew rate, the excitation k-space trajectories extend beyond the spokes along the $k_{z}$ direction. [Color figure can be viewed in the online issue, which is available at wileyonlinelibrary.com.]

on the reduction in the SAR. An NLGF that shows higher compatibility with the desired excitation profile than the field used in the example may yield a higher reduction in the SAR. With the implementation of the VERSE algorithm, the SAR of both the LGF and NLGF cases was reduced. However, with VERSE implementation, the SAR of the nonlinear gradient case was nearly $40 \%$ lower than that of the linear case in all demonstrated cases.

In the third example, a gradient coil with a diameter of $16 \mathrm{~cm}$ was used with a maximum current of 5 A (4 turns $\times 1.25 \mathrm{~A}$ ). Although the size and the low-current requirement hint at the applicability of the proposed technique 


\section{Linear}

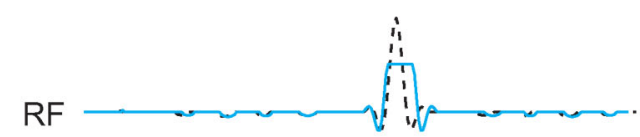

Nonlinear

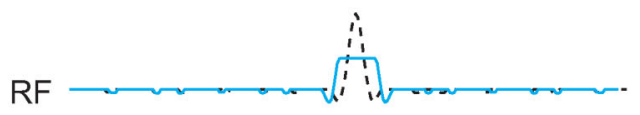

\section{$10 \%$}
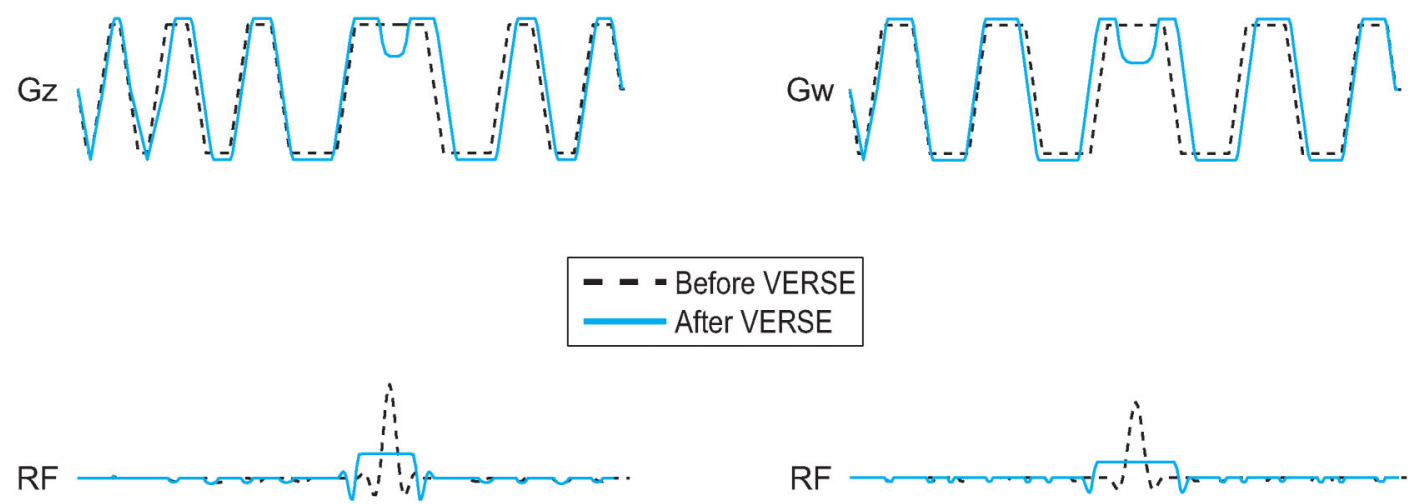

$25 \%$

\section{Slew Rate Limited $(100 \%)$}
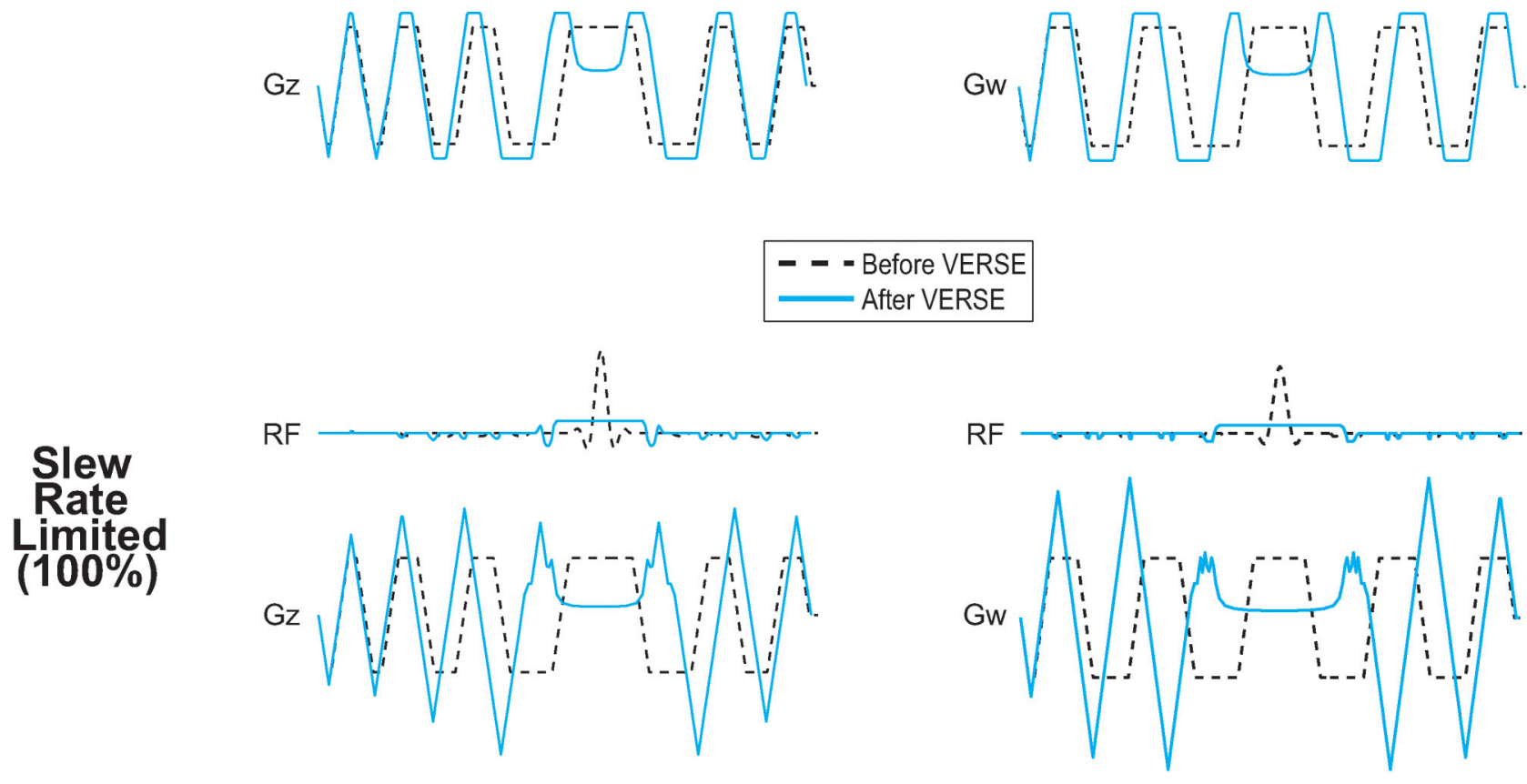

FIG. 9. Comparison of the RF pulses for the linear and nonlinear cases when the VERSE algorithm is implemented. To implement the VERSE algorithm, 10, 25, and $100 \%$ increases in the maximum gradient amplitude are allowed. [Color figure can be viewed in the online issue, which is available at wileyonlinelibrary.com.]

to extremity and head imaging with slightly larger dimensions, the extension of the approach to whole-body MRI needs further investigation because it is known that the power demands from the gradient amplifiers scale with the fifth power of the coil diameter (27). However, in vivo MRI applications of NLGFs have been used previously for encoding during head MRI (28).

When NLGFs are used, the reduction in the SAR depends on the compatibility of the NLGFs and the desired excitation profile. Therefore, the optimal field may vary for different applications. However, sometimes, the optimal NLGF may be too demanding from a hardware standpoint, or it may require impractical coil dimensions. Therefore, a trade-off exists between the SAR reduction and the feasibility of achieving that reduction.

Because the SAR depends on the compatibility of the shape, position, orientation, and thickness of the excitation profile, certain NLGFs may increase the SAR, as well. In 
such a case, if the LGFs are still available along with the NLGFs, the SAR-optimal pulse would use only the LGFs, preventing any increase in SAR. Therefore, when NLGFs are available along with some linear counterparts, any excitation profile can be obtained, and the SAR may only be lower than the linear case.

The use of NLGFs alters the well-known Fourier transform relationship that exists between the excitation profile and the excitation k-space in the small-tip-angle regime. When NLGFs are used, the Larmor frequency of spins varies nonlinearly in space, and the kernel of the Fourier transform becomes a function of the nonlinear field distribution. In this case, RF pulses can be designed with NLGFs either with this nonlinear Fourier transform or by defining a nonlinear coordinate system to obtain the well-known linear form of the Fourier transform. Because the kernel changes (in the former approach) or the excitation profile changes (in the latter approach), the excitation k-space and the RF pulse are altered, leading to the change in the SAR. It should be noted that similar approaches can be applied at the large-tip-angle regime, as well. For example, NLGFs can be used for designing large-tip-angle pulses with the Cayley-Klein parameterization algorithm (29) by expressing the excitation profile in a nonlinear coordinate system or incorporating the nonlinear field variation in the CayleyKlein parameters. However, further investigation of the applicability of this method is needed.

The optimization of MRI and its applications is a complex problem with many design goals, including the reduction of the SAR, the reduction of the scan time, the increase of the resolution, the increase of the signal-to-noise ratio and the increase of the field homogeneity. We believe that by combining various approaches to solving this problem, such as $B_{1}^{+}$-shimming, multidimensional excitation pulses, variable-rate selective excitation, encoding using NLGFs, and coil design, significantly different imaging schemes can be designed that not only better suit the region of interest or the goal of examination but also increase the efficiency in terms of imaging time, global SAR, and local SAR.

\section{REFERENCES}

1. Guidance for the Submission of Premarket Notifications for Magnetic Resonance Diagnostic Devices, Tech. Rep., Food and Drug Administration, 1998.

2. I. E. Commission. Medical electrical equipment. Part 2. Particular requirements for the safety of magnetic resonance equipment for medical diagnosis, International Standard, Vol. 60601; 2002. pp 2-33.

3. Kopanoglu E, Erturk VB, Atalar E. Analytic expressions for the ultimate intrinsic signal-to-noise ratio and ultimate intrinsic specific absorption rate in MRI. Magn Reson Med 2011;66:846-858.

4. Metzger GJ, Snyder C, Akgun C, Vaughan T, Ugurbil K, de Moortele PFV. Local B1+ shimming for prostate imaging with transceiver arrays at 7T based on subject-dependent transmit phase measurements. Magn Reson Med 2008;59:396-409.

5. Poser BA, Koopmans PJ, Witzel T, Wald LL, Barth M. Three dimensional echo-planar imaging at 7 Tesla. Neuroimage 2010;51:261-266.

6. Zelinski AC, Wald LL, Setsompop K, Goyal VK, Adalsteinsson E. Sparsity-enforced slice-selective MRI RF excitation pulse design. IEEE Trans Med Imaging 2008;27:1213-1229.
7. Zelinski AC, Wald LL, Setsompop K, Alagappan V, Gagoski BA, Goyal VK, Adalsteinsson E. Fast slice-selective radio-frequency excitation pulses for mitigating $\mathrm{B}+1$ inhomogeneity in the human brain at 7 Tesla. Magn Reson Med 2008;59:1355-1364.

8. Setsompop K, Alagappan V, Gagoski B, Witzel T, et al. Slice-selective rf pulses for in vivo b1+ inhomogeneity mitigation at 7 tesla using parallel rf excitation with a 16-element coil. Magn Reson Med 2008;60:14221432.

9. Yang C, Deng W, Alagappan V, Wald LL, Stenger VA. Four-dimensional spectral-spatial RF pulses for simultaneous correction of B1+ inhomogeneity and susceptibility artifacts in T2*-weighted MRI. Magn Reson Med 2010;64:1-8.

10. Bottomley PA, Charles HC, Roemer PB, Flamig D, Engeseth H, Edelstein WA, Mueller OM. Human in vivo phosphate metabolite imaging with 31P NMR. Magn Reson Med 1988;7:319-336.

11. Conolly S, Nishimura D, Macovski A, Glover G. Variable-rate selective excitation. J Magn Reson 1988;78:440-458.

12. Wilm BJ, Barmet C, Pavan M, Pruessmann KP. Higher order reconstruction for MRI in the presence of spatiotemporal field perturbations. Magn Reson Med 2011;65:1690-1701.

13. Schneider JT, Haas M, Ruhm W, Hennig J, Ullmann P. Robust spatially selective excitation using radiofrequency pulses adapted to the effective spatially encoding magnetic fields. Magn Reson Med 2011;65:409-421.

14. Lee SY, Cho ZH. Localized volume selection technique using an additional radial gradient coil. Magn Reson Med 1989;12:56-63.

15. Oh CH, Hilal SK, Cho ZH, Mun IK. New spatial localization method using pulsed high-order field gradients (SHOT: Selection with HighOrder gradienT). Magn Reson Med 1991;18:63-70.

16. Wu EX, Johnson G, Hilal SK, Cho ZH. A new 3D localization technique using quadratic field gradients. Magn Reson Med 1994;32:242-245.

17. Weber H, Gallichan D, Schultz G, Witschey WR, Welz AM, Cocosco CA, Hennig J, Zaitsev M. ExLoc: excitation and encoding of curved slices. In Proceedings of the 19th Annual Meeting of ISMRM, Montreal, Canada, 2011. p. 2806.

18. Hennig J, Welz AM, Schultz G, Korvink J, Liu Z, Speck O, Zaitsev M. Parallel imaging in non-bijective, curvilinear magnetic field gradients: a concept study. MAGMA 2008;21:5-14.

19. Schultz G, Ullmann P, Lehr H, Welz AM, Hennig J, Zaitsev M. Reconstruction of MRI data encoded with arbitrarily shaped, curvilinear, nonbijective magnetic fields. Magn Reson Med 2010;64:1390-1403.

20. Gallichan D, Cocosco CA, Dewdney A, Schultz G, Welz A, Hennig J, Zaitsev M. Simultaneously driven linear and nonlinear spatial encoding fields in MRI. Magn Reson Med 2011;65:702-714.

21. Haas M, Ullmann P, Schneider JT, Ruhm W, Hennig J, Zaitsev M. Large tip angle parallel excitation using nonlinear non-bijective PatLoc encoding fields. In Proceedings of the 18th Annual Meeting of ISMRM, Stockholm, Sweden, 2010. p. 4929.

22. Stockmann JP, Ciris PA, Galiana G, Tam L, Constable RT. O-space imaging: highly efficient parallel imaging using second-order nonlinear fields as encoding gradients with no phase encoding. Magn Reson Med 2010;64:447-456.

23. Marro KI, Lee D, Hyyti OM, Nonlinear magnetic field gradients can reduce sar in flow-driven arterial spin labeling measurements. J Magn Reson 2007;185:94-102.

24. Kopanoglu E, Akin B, Erturk V, Atalar E. SAR Reduction using nonlinear gradients. In Proceedings of the 19th Annual Meeting of ISMRM, Montreal, Canada, 2011. p. 1848.

25. Pauly J, Nishimura D, Macovski A. A k-space analysis of small-tip-angle excitation. J Magn Reson 1989;81:43-56.

26. Turner R. A target field approach to optimal coil design. J Phys D: Appl Phys 1986;19:147-151.

27. Bowtell R, Mansfield P. Gradient coil design using active magnetic screening. Magn Reson Med 1991;17:15-9; discussion 19-21.

28. Cocosco CA, Gallichan D, Dewdney AJ, Schultz G, Welz AM, Witschey WR, Weber H, Hennig J, Zaitsev M. First in-vivo results with a PatLoc gradient insert coil for human head imaging. In Proceedings of the 18th Annual Meeting of ISMRM, Stockholm, Sweden, 2010. p. 4929.

29. Pauly J, Nishimura D, Macovski A. A linear class of large-tip-angle selective excitation pulses. J Magn Reson 1989;82:571-587. 